\title{
ON GRADED RINGS WITH FINITENESS CONDITIONS
}

\author{
V. P. CAMILLO AND K. R. FULLER
}

\begin{abstract}
It is proved that a graded ring that is finitely graded modulo its radical is local if its initial subring is local, and that a graded artinian ring is finitely generated over its initial subring which is also artinian. These results extend theorems of Gordon and Green on artin algebras. Other results relating the structure of a graded ring to that of its initial subring are also presented.
\end{abstract}

In this note we provide simple proofs of extensions of the principal results in $\mathbf{R}$. Gordon and E. Green's recent paper [4] on graded artin algebras. First we prove that any finitely graded (modulo the radical) ring with local initial subring is itself local. From this it follows that over any graded ring a finitely graded module with a composition series is an indecomposable module if and only if it is indecomposable as a graded module. Then we prove that a graded ring is left artinian if and only if it has a composition series as a left module over its initial subring. These results are used to show that over a graded left artinian ring every simple module, every projective module, and every injective left module is isomorphic to a graded module, as is every direct summand of a finitely generated graded left module. Also they yield information about the relative structure of a graded ring and its initial subring, for example, a finitely graded ring is semiprimary if and only if so is its initial subring.

Recall (see [3, 6]) that a (Z-) graded ring is a ring $R$ together with an abelian group decomposition $R=\bigoplus_{\mathrm{Z}} R_{n}$ such that $R_{n} R_{m} \subseteq R_{n+m}(n, m \in \mathbf{Z})$, and that $R_{0}$ is a unital subring called the initial subring of $R$. We let $J=J(R)$ denote the radical of $R$ and $J_{0}=J\left(R_{0}\right)$. Our first result, generalizing [4, Theorem 3.1], requires no more than this.

1. TheOREM. Let $R=\bigoplus_{\mathrm{Z}} R_{n}$ be a graded ring. If $R_{0}$ is local and $R_{n} \subseteq J$ for all but finitely many $n \in \mathbf{Z}$, then $R$ is a local ring.

Proof. By hypothesis there is an $N \geqslant 0$ such that $R_{n} \subseteq J$ whenever $|n|>N$. Thus each $R_{n}$ with $n \neq 0$ consists of zero divisors modulo $J$, and it follows that $R_{n} R_{-n}$ contains no units. Therefore, since $R_{0}$ is local, $R_{n} R_{-n} \subseteq J_{0}$ whenever $n \neq 0$. Let $k>0$ and suppose, inductively, that $R_{n} \subseteq J$ whenever $|n|>k$. Let $b_{k} \in R_{k}, a=$ $\sum a_{n} \in R$, and $x=1-b_{k} a$. To see that $b_{k} \in J$ we show that $x$ is invertible. Since

Received by the editors September $8,1981$.

1980 Mathematics Subject Classification. Primary 16A03, 16A35; Secondary 16A10, 16A51. 
$|n-k|>k$ when $n<0$,

$$
\begin{aligned}
x & =1-\sum_{n<0} b_{k} a_{n-k}-b_{k} a_{-k}-\sum_{n>0} b_{k} a_{n-k} \\
& =1-j-j_{0}-p
\end{aligned}
$$

with $j \in J, j_{0} \in J_{0}$ and $p \in \Sigma_{n>0} R_{n}$. Let $u_{0}=\left(1-j_{0}\right)^{-1} \in R_{0}$. Then

$$
x u_{0}=1-j^{\prime}-p^{\prime}
$$

with $j^{\prime} \in J$ and $p^{\prime} \in \Sigma_{n>0} R_{n}$. But then $p^{\prime}$ is nilpotent modulo $J$ and so $\left(1-p^{\prime}\right)^{-1}=v$ exists in $R$. Now we have

$$
x u_{0} v=1-j^{\prime \prime}
$$

with $j^{\prime \prime} \in J$ so $x u_{0} v$, and hence $x$, is (right) invertible. Thus $R_{k} \subseteq J$, and similarly $R_{-k} \subseteq J$, and we have shown that $R_{n} \subseteq J$ whenever $n \neq 0$. It follows that $r_{0} \mapsto r_{0}+J$ defines a surjective ring homomorphism $R_{0} \rightarrow R / J$ so $R$ is local.

A left $R$-module $M$ is graded $[3,6]$ in case it has a decomposition $M=\bigoplus_{\mathrm{z}} M_{n}$ over $R_{0}$ such that $R_{m} M_{n} \subseteq M_{m+n}(m, n \in \mathbf{Z})$. If $N=\bigoplus_{\mathrm{Z}} N_{n}$ is also a graded module, the elements of $\operatorname{Hom}_{R}(M, N)_{n}=\left\{f \in \operatorname{Hom}_{R}(M, N) \mid f\left(M_{k}\right) \subseteq N_{k+n}(k \in\right.$ Z)\} are called degree $n$ homomorphisms. The category $R-G r$ consisting of graded left $R$-modules and degree zero homomorphisms is a Grothendieck category [5]. The images (kernels) of degree zero homomorphisms to (from) $M$ are the homogeneous submodules of $M$. If all but finitely many terms in the grading of a graded module or ring are zero then it is said to be finitely graded. Now Theorem 1 allows us to free [4, Theorem 3.2] of any finiteness conditions on its graded ground ring.

2. Corollary. Let $M$ be a finitely graded R-module with a.c.c. and d.c.c. on homogeneous submodules. Then $M$ is indecomposable in $R-\mathrm{Gr}$ if and only if $M$ is indecomposable in R-Mod.

Proof. One implication is obvious. For the other let $S=\operatorname{End}\left({ }_{R} M\right)$. Then $S$ is finitely graded with initial subring $S_{0}$ (see [5, Lemma 3.3.2, p. 83]), the ring of zero degree endomorphisms of $M$. If $s_{0} \in S_{0}$ then by Fitting's Lemma $M=\operatorname{Im} s_{0}^{c} \oplus \operatorname{Ker} s_{0}^{c}$ where $c$ is the composition length of $M$. But these are homogeneous submodules of $M$, so $s_{0}$ is invertible or nilpotent. Thus $S_{0}$ is local and so, by Theorem 1 , is $S$.

According to [6, Lemma 3.3.6, p. 9] if any two maps in an equation $f=g h$ of $R$-maps between graded $R$-modules are of degree zero, then the third can be replaced by a degree zero homomorphism. It follows that any homogeneous $R$-direct summand of a graded $R$-module has at least one homogeneous complement, and that graded projective (injective) (semisimple) $R$-modules are projective (injective) (semisimple) in $R-\mathrm{Gr}$.

Let $M=\oplus_{\mathrm{Z}} M_{n}$ be a graded left $R$-module and let $k \in \mathbf{Z}$. For each $R_{0}$-submodule $N_{k} \leqslant R_{0} M_{k}$ let $\theta_{k}\left(N_{k}\right)=R N_{k}$, and for each homogeneous submodule $N=\bigoplus_{\mathrm{z}} N_{n}$ of $M$ let $\varphi_{k}(N)=N_{k}$. Then $\theta_{k}$ and $\varphi_{k}$ define order preserving maps between the lattices of $R_{0}$ submodules of $M_{k}$ and homogeneous submodules of $M$. 
3. LEMMA. The order preserving maps $\theta_{k}$ and $\varphi_{k}$ satisfy

$$
\varphi_{k} \theta_{k}\left(N_{k}\right)=N_{k} \quad\left(N_{k} \leqslant R_{0} M_{k}\right) .
$$

Moreover $\varphi_{k}(N)$ is a direct summand of $M_{k}$ whenever $N$ is a homogeneous direct summand of $M$.

Proof. If $N_{k} \leqslant R_{0} M_{k}$ then $\theta_{k}\left(N_{k}\right)=R N_{k}=\bigoplus_{n \in \mathrm{Z}} R_{n-k} N_{k}$ and $\varphi_{k} \theta_{k}\left(N_{k}\right)=R_{0} N_{k}$ $=N_{k}$. If $N=\bigoplus_{\mathrm{z}} N_{n}$ is a homogeneous direct summand of $M$ then $N$ has a homogeneous complement $N^{\prime}=\oplus N_{n}^{\prime}$. But then clearly $M_{k}=\varphi_{k}\left(N \oplus N^{\prime}\right)=N_{k} \oplus$ $N_{k}^{\prime}$.

When discussing kinds of graded rings, our other descriptive adjectives apply directly to the ring in question, irregardless of its grading. Thus a graded semisimple ring is a semisimple ring that happens to be graded, and graded division rings are trivially graded, i.e., $R=R_{0}$ (see [6, Lemma 6.1, p. 58]). The following results show that, up to automorphism, semisimple rings can only be graded in finitely many ways.

4. Proposition. If $R=\bigoplus_{\mathrm{Z}} R_{n}$ is a graded semisimple ring then $R_{0}$ is semisimple and contains a complete orthogonal set $e_{1}, \ldots, e_{m}$ of primitive idempotents of $R$ such that for all $i, j \in\{1, \ldots, m\}$ there is an $n \in \mathbf{Z}$ with $e_{i} R_{j} \subseteq R_{n}$.

Proof. First assume that $R_{0}$ is a division ring. Since semisimple rings are von Neumann regular, if $0 \neq v_{n} \in R_{n}$ then there is an $a=\sum a_{i} \in R$ such that

$$
r_{n}=r_{n} a r_{n}=\sum_{i} r_{n} a_{i-2 n} r_{n}
$$

so we have $r_{n}=r_{n} a_{-n} r_{n}$. But then, since $a_{-n} r_{n} \in R_{0}$, a division ring, and $r_{n} \neq 0$ we see that $a_{-n} r_{n}=1$. Thus every nonzero homogeneous element of $R$ is invertible, and this implies that $R$ is a (noncommutative) domain. Thus $R$ is a division ring and $R=R_{0}$.

Now suppose that $R$ is semisimple. Then so, by Lemma 3 , is $R_{0}$. Let $e_{1}, \ldots, e_{m}$ be a complete set of orthogonal primitive idempotents of $R_{0}$. Then $e_{i} R_{0} e_{i}$, the initial subring of the graded semisimple ring $e_{i} R e_{i}=\bigoplus_{n} e_{i} R_{n} e_{i}$, is a division ring, so

$$
e_{i} R_{i}=e_{i} R_{0} e_{i} \subseteq R_{0}
$$

for $i=1, \ldots, m$. Next let $e_{i} a e_{j} \in e_{i} \operatorname{Re}_{j}$ and write

$$
e_{i} a e_{j}=\sum_{k} a_{k}=\sum_{k} e_{i} a_{k} e_{j}
$$

If some $e_{i} a_{n} e_{j} \neq 0$ then, since $e_{i} R e_{j}$ is one-dimensional over $e_{i} R e_{i}=e_{i} R_{0} e_{i}$, for each $k \in \mathbf{Z}$ there is an $e_{i} b_{0} e_{i} \in e_{i} R_{0} e_{i}$ such that

$$
e_{i} a_{k} e_{j}=e_{i} b_{0} e_{i} a_{n} e_{j} \in R_{n} .
$$

But then $e_{i} R e_{j} \subseteq R_{n}$, and the proof is complete.

Now employing G. Bergman's recent theorem [2]: The Jacobson radical of a graded ring $R$ is a homogeneous ideal of $R$ (which incidentally proves the converse of 
Theorem 1), we can easily prove

5. TheOREM. A graded ring $R=\bigoplus_{\mathrm{Z}} R_{n}$ is left artinian if and only if $R_{0}$ is left artinian and $R$ is finitely generated as a left $R_{0}$-module.

Proof. Let $R$ be left artinian. By Proposition 4, $R / J=\oplus_{\mathrm{z}} R_{n}+J / J$ is finitely graded, so by Lemma $3 R / J$ has a composition series over $R_{0}+J / J$. Thus every simple $R$-module, and hence $R$, has a composition series over $R_{0}$. The converse is obvious.

This theorem generalizes [4, Lemma 1.3] from the commutative case and provides an effective analogue to [4, Theorem 1.4] by showing that graded finitely generated modules over a graded artinian ring are finitely graded. Thus by Theorems 2 and 5 we have

6. Corollary. Let $R$ be a graded left artinian ring. A graded finitely generated $R$-module is indecomposable in $R-\mathrm{Gr}$ if and only if it is indecomposable in $R$-Mod.

Gordon and Green call an $R$-module gradable in case it is $R$-isomorphic to a module in $R-\mathrm{Gr}$. The list of gradable modules over a graded artin algebra, given in $[4, \S 3]$, is also valid in the left artinian case.

7. Proposition. If $R$ is a graded left artinian ring then every left $R$-module that is semisimple, projective or injective is gradable, and so are all direct summands of finitely generated gradable modules.

Proof. The last statement follows from Corollary 6 and the Krull-Schmidt Theorem; and it implies that finitely generated, hence all, semisimple and projective left $R$-modules are gradable.

To see that injective modules are gradable, suppose $R$ is graded $R=\bigoplus_{-N}^{N} R_{n}$, let $C_{0}$ be an injective cogenerator in $R_{0}$-Mod, and let

$$
C=\operatorname{Hom}_{R_{0}}\left(R_{R}, C_{0}\right)=\bigoplus_{-N}^{N} \operatorname{Hom}_{R_{0}}\left(R_{-n}, C_{0}\right)
$$

to obtain a finitely graded injective cogenerator $C$ [1, Exercises 19.14, 19.20] in $R$-Mod. Now we note that $\operatorname{Soc}_{R} C=\operatorname{ann}_{C}(J)$ is a homogeneous submodule of $C$ that is semisimple and essential in $C$ as an object of $R-\mathrm{Gr}$, that simple objects in $R$-Gr are simple in $R$-Mod (i.e., isomorphic to one of the $R e_{i}$ of Proposition 4), and we observe that a minor modification of the usual proof in $R$-Mod shows that, since $R$ has a.c.c. on homogeneous left ideals, direct sums of injectives are injective in $R$-Gr. Using these facts we have $C=\amalg E\left(S_{\alpha}\right)$ in $R-\mathrm{Gr}$, where $S_{\alpha}$ are $R$-simple homogeneous submodules of $C$ such that $\operatorname{Soc} C=\amalg S_{\alpha}$ in $R$-Mod. But then, since $C$ is a cogenerator, the injective envelope of each simple left $R$-module must be isomorphic to one of the $E\left(S_{\alpha}\right)$, and so every injective left $R$-module must be gradable.

Most of the remaining results on graded artin algebras of Gordon and Green's [4 and 5] can now be shown to hold for graded left artinian rings, with only minor 
adjustments in their arguments. (For example, injectives in $R-G r$ are injective as $R$-modules.) However, their ultimate theorems $[5, \S \S 3,4]$, showing that every module is gradable over a graded artin algebra of finite type, and that a graded artin algebra has finite type if and only if its category of gradable modules does, have proofs relying heavily on the Auslander-Reiten "dual of the transpose" which is defined only for artin algebras; there are no alternative arguments apparent to us.

We conclude with some applications relating the structure of $R$ to that of $R_{0}$. If $R$ is a semilocal graded ring, then by Proposition 4 the grading $R / J=\bigoplus R_{n}+J / J$ is finite and $R_{0} / J \cap R_{0} \cong R_{0}+J / J$ is semisimple. It follows from the latter that $J \cap R_{0}=J_{0}$ (since always $J \cap R_{0} \subseteq J_{0}$ ). Thus

8. Proposition. If $R$ is semilocal, semiprimary, left perfect, or semiperfect with nil radical, then so is $R_{0}$ and $R / J$ is finitely graded.

On the other hand it follows from Theorem 1 and [1, Theorem 27.6] that

9. Proposition. If $R_{0}$ is semiperfect and $R / J$ is finitely graded then $R$ is semiperfect and has a complete set of primitive idempotents of degree zero; and so every projective $R$-module is gradable.

We do not know whether $R$ semiperfect implies $R_{0}$ semiperfect, but from the above propositions and minor modifications of the nilpotency argument of $[4$, Theorem 3.1] one can deduce that

10. Proposition. A finitely graded ring is semiprimary or left or right perfect if and only if its initial subring is.

This is not the case for artinian rings as the ring of real matrices

$$
\left[\begin{array}{ll}
\mathbf{Q} & \mathbf{R} \\
0 & \mathbf{Q}
\end{array}\right]
$$

clearly shows.

\section{REFERENCES}

1. F. W. Anderson and K. R. Fuller, Rings and categories of modules, Springer-Verlag, New York and Berlin, 1974.

2. G. Bergman, On Jacobson radicals of graded rings, preprint.

3. R. Fossum and J. B. Foxby, The category of graded modules, Math. Scand. 34 (1975), 288-300.

4. R. Gordon and E. L. Green, Graded artin algebras, preprint.

5. $\longrightarrow$, Representation theory of graded artin algebras, preprint.

6. C. Năstăsescu and F. Van Ozotaeyen, Graded and filtered rings and modules, Lecture Notes in Math., vol. 758, Springer-Verlag, Berlin and New York, 1979.

Department of Mathematics, The University of Iowa, Iowa City, Iowa 52242 